\title{
$\mathrm{HCl}$ at End of Life \& Beyond
}

\section{Jayne Wallace}

Northumbria University

Newcastle upon Tyne, UK

jayne.wallace@northumbria.ac.uk

\section{Will Odom}

Simon Fraser University

Surrey, B.C., Canada

wodom@sfu.ca

\section{Kyle Montague}

Newcastle University

Newcastle upon Tyne, UK

kyle.montague@newcastle.ac.uk

\section{Nantia Koulidou}

Northumbria University

Newcastle upon Tyne, UK

nantia.koulidou@northumbria.ac.uk

\section{Corina Sas}

Lancaster University

Lancaster, UK

c.sas@lancaster.ac.uk

\section{Kellie Morrissey}

University of Limerick

Limerick, Ireland

kellie.morrissey@ul.ie

\section{Patrick Olivier}

Monash University

Melbourne, Australia

patrick.olivier@monash.edu

Permission to make digital or hard copies of part or all of this work for personal or classroom use is granted without fee provided that copies are not made or distributed for profit or commercial advantage and that copies bear this notice and the full citation on the first page. Copyrights for third-party components of this work must be honored. For all other uses, contact the owner/author(s).

CHI 2020 Extended Abstracts, April 25-30, 2020, Honolulu, HI, USA. (C) 2020 Copyright is held by the owner/author(s)

ACM ISBN 978-1-4503-6819-3/20/04

DOI: https://doi.org/10.1145/3334480.XXXXXXX

*update the above block \& DOI per your rightsreview confirmation (provided after acceptance)

\begin{abstract}
Death, whilst an inevitable part of being alive, factors more significantly in our lives than the event itself. The role that technology can play in how people live as they approach end of life as well as in bereavement is full of rich possibilities, but research here is also fraught with ethical and methodological dilemmas. Although there has been a turn to focus on the topic of death by some in HCI we need to go far further to embrace the contexts relating to it more meaningfully and broadly. Through this design focused workshop, we will bring experts and interested parties together to creatively explore opportunities and challenges for $\mathrm{HCI}$ at the end of life and beyond. Discussions and design activities will be supported by conceptual resources for design, lived experience accounts, design methods and ethical resources. The workshop will provide a time and place to bring together experts but will also provide an open and accepting environment for those for whom $\mathrm{HCI}$ at end of life and beyond is a new area of concern.
\end{abstract}

\section{Author Keywords}

Death, dying, end of life, bereavement, continuing bonds, ethics, design.

\section{CSS Concepts}

Human-centered computing; Human computer interaction ( $\mathrm{HCI}$ ); HCI theory, concepts and models 


\section{Motivation}

Death is an inevitable part of being alive and involves a wide set of related practices that vary across cultures.

Whilst death is in itself an event, both approaching end of life and grieving occur in the social sphere and

involve experiences and practices that span a range of timeframes and relational forms of social

connectedness. Whilst HCI has paid the context of death some attention in recent years (to a large extent stimulated by Massimi et al's CHI 2010 workshop [8] and paper [9]) active programs of research in this area remain in short supply.

End of life and beyond has implication for almost all users of digital technologies, from the expression of future wishes and bequeathing of assets, to personal archiving and memorialisation of those who have died through digital content. Mainstream technology providers may have policies in place to address the eventualities the death of "customers", however it is hard to imagine that end of life has in any way been part of the blueprint of the service design. Indeed, the lack of engagement of $\mathrm{HCI}$ and design researchers with 'matters of life and death' is mirrored by mainstream digital products and services, which largely ignore the deeper needs of people in relation to this crucial element of their personal and social lives.

While Massimi et al's ground-breaking foray sought to articulate an $\mathrm{HCI}$ research for end of life by first mapping "questions concerning materiality and artefacts, social identities, temporality and methodologies", their proposals for a design agenda were notably limited. Yet developments in technologies in the eight years that have followed (particularly in relation to algorithmic interaction and personal media) point to a far richer space for designing digital technologies for of end of life and beyond. Indeed, we are living in an age with unforeseen capabilities to make both physical and digital "things" and where each person's life has an unavoidable associated trail of media and personal data. New opportunities to curate data and media to support others after our future death or to support ourselves in bereavement are substantial $[14,18]$ and the contexts of anticipating end of life and living with bereavement are changing as digital technologies become more embedded in our cultures $[13,15]$. While it is easy to recognize that these opportunities for design are significant, and that the need is substantial and largely unaddressed, the barriers to working in a such sensitive context are perceived by many $\mathrm{HCI}$ researchers as overly daunting. Working with people around topics of death, dying and bereavement requires both a level of sensitivity and self-reflection that will be new, and even intimidating, to many researchers. It is also an area that requires the examination of unfamiliar conceptual resources and new methods for both design and evaluation.

Our contention is that the end of life, and beyond, is such an important aspect of personal and social experience that $\mathrm{HCI}$ has a responsibility to engage with it. On the one hand, we see this workshop as a rallying call to $\mathrm{HCI}$ and design researchers who are seeking to respond to the challenges of this sensitive domain. On the other hand, the workshop is an invitation to those in the wider $\mathrm{HCI}$ and design community with lived experience of death, dying and bereavement, who are prepared to step outside their familiar domains of expertise. Thus, the workshop will provide a time and place to bring together experts but will also provide an open and accepting environment for those for whom $\mathrm{HCI}$ at end of life and beyond is a new area of concern. 


\section{Themes}

We will organize both the call for participation and the workshop activities around three themes related to $\mathrm{HCI}$ research at end of life and beyond. These themes will both clarify our goals and support participants -

experts and non-experts alike - in their preparations for the workshop discussions and participation in design activities. Note, the workshop activities will necessarily assume high levels of pre-event engagement and we will make this clear in both our call for participation and our notification of acceptance of attendance.

\section{Theme 1: Conceptual Resources for Design}

Theories of bereavement are at the same time one of the most useful, and also one the least familiar, resources for $\mathrm{HCI}$ researchers new to this space. For this reason, and without excluding alternative or complementary positions, we will be explicit about the theories and frameworks of loss and bereavement that we will be asking participants to engage with: (i) stages \& phases; (ii) dual-process theory; and (iii) continuing bonds. To this end we will be providing potential attendees (both pre-submission and post-acceptance) with a set of curated resources that help them to familiarize themselves with these theories.

(i) Stages \& Phases: In the West since Freud [3], dominant grieving and mourning practices have been conceived as the processes whereby the bereaved person adjusts to the reality of their loss, enabling them to disengage from the deceased and reinvest in new relationships. Numerous theories that have followed have broadly been based on the notion that the process of bereavement follows a set of stages whereby the bereaved moves between phases as feels right for them personally. Kübler-Ross [7] 5 stages of grief (denial, anger, bargaining, depression, acceptance) describes how people cope with illness and was only later extended to grieving. Bowlby and Parkes [2] applied Bowlby's attachment theory to present four main stages in the grief process. While Kübler-Ross's work was largely based on clinical observations, Bowlby and Parkes applied attachment theory in characterising grieving not as a state (stages) but as a process (phases) that the bereaved needed to work through. More pragmatic approaches, such as Worden's [19], frames mourning in terms of active 'grief work' to be undertaken to move beyond the passive phases of grief: (i) to accept the reality of loss; (ii) to work through and experience the pain of grief; (iii) to adjust to an environment without the deceased person; and (iv) to withdraw emotionally from or relocate the deceased and move on with life.

(ii) The Dual-Process Model: Although stages \& phases approaches are the most widely known and accepted theories and frameworks of bereavement, on which most contemporary therapies and self-help guidance is based, they are not universally accepted. Indeed, Stroebe and Schut [16] critiqued the stages \& phases view for its linear characterisation of grieving, prescriptive nature, narrowly western perspective, tendency to oversimplify the complex phenomena of loss particularly in relation to individual differences, and lack of empirical validation. Instead, Stroebe \& Schut draw on Cognitive Stress Theory in presenting their "Dual-Process Theory", an alternative view of how people come to terms with the bereavement of a person close to them. In their alternative model of "coping" they identify two classes of 'stressors', loss and restoration. In the loss-oriented process the bereaved engages with the recognition and acceptance 
of the loss itself, associated changes personal, social and economic circumstances, and their own identity. In the restoration process, the bereaved focuses on new aspects of their post-loss reality, that is, issues that need to be addressed and how to address them. Stroebe \& Schut propose a "dynamic, regulatory coping process of oscillation, whereby the grieving individual at times confronts, at other times avoids, the different tasks of grieving" [16 p. 197].

(iii) Continuing Bonds. An alternative approach that focuses on continued connections with deceased persons, rather than detachment, have become prevalent in the West over the last two decades and have brought a return to pre-modernist Western practices. Klass, Silverman and Nickman's [5, 6] notion of continuing bonds articulates a concept of grief that acknowledges the value of a continued sense of connection between the bereaved and the deceased. Rather than seeing grief as a process working towards 'letting go' they advocate processes whereby people find ways to sustain the presence of the deceased in their lives in order to find healthy ways to live with bereavement. There is a fundamental recognition firstly that people are relational selves wherein sense of self is supported by others and secondly that this does not end when a loved one dies. There are social and cultural precedents for such ongoing relationships with the dead within many non-western cultures, including Maori practices and the Marae [11] and the Sora of Eastern India [17]. As such a continued connection to the dead is nothing new but is something that in the West we lost in the 20th Century "marginalized by the discourses and practices of modernity" [4 p.127].
Theme 2: Design Methods for End of Life Research Enabling people to engage in conversations about emotionally loaded content around death, dying and bereavement is challenging for researchers. There is the opportunity to focus on ways to conduct research in these sensitive contexts which are underrepresented in HCI research. Design will be explored through the introduction of readily accessible methods such as Blueprints and Life Cafe, which will be introduced as starting points to discuss how to facilitate participatory engagements for this challenging context. Design can offer sensitive methods that are responsible to the context and result in appropriate forms of knowledge for HCI. In linking to technology, we will consider the appropriation of digital media to support people by offering meaningful interactions in the contexts of bereavement and anticipation of death. Topics of interest include (but are not limited to): designing with metadata, designing with digital services/platforms, and augmenting digital and physical objects.

Understanding how to design platforms and tools for meaningful experiences in interacting with digital objects and services for people who are bereaved will be a major element of this theme in the workshop.

Theme 3: Ethical Issues with End of Life Research Ethics are a system of moral principles and branch of knowledge enquiry defining what is good for individuals and society. Whilst academic disciplines operate within publicly defined ethical parameters, both ethical codes and procedures can be protectionist, stifle creativity and focus more on process than people. This has led to calls for a more situated ethics and an acceptance that this is a good way forward. The last five years has seen a growing interest in ethics from within the $\mathrm{HCI}$ community as evidenced by plethora of papers and 


\section{Website}

The workshop website will be hosted at:

http://enablingongoingess.co $\mathrm{m} / \mathrm{chi} 2020$

We will display the call for participation, deadlines and accepted submissions.

After the workshop we will update the webpage to reflect the workshop outcomes and make materials available to the $\mathrm{CHI}$ community. We will continue to use the page as a basis for discussion and community building activities. growing number of workshops $[1,10,12]$. As researchers increasingly work in interdisciplinary teams within the context of health and wellbeing, they are being required to navigate unfamiliar ethical contexts and research dilemmas. Within the workshop we will weave ethics discussions pertinent to interdisciplinary working as well as the contexts of approaching end of life and bereavement into the design activities using a range of resources to support this.

\section{Organizers}

Jayne Wallace (Northumbria University, UK) is Professor of Craft and Wellbeing in the School of Design, Northumbria University. Her research explores the potential of design, contemporary jewellery, digital technologies, and cocreative acts of making to support sense of self across a range of complex health and social care contexts. Particular areas of interest include anticipating end of life, ongoingness in bereavement and personhood in dementia. She co-founded the Research Through Design conference series, is on the editorial board for the Design for Health Journal and cofounded the Journal for Jewellery Research. She is currently PI on the RCUK Enabling Ongoingness project.

Corina Sas (Lancaster University, UK) is Professor in Human-Computer Interaction and Digital Health in the Department of Computing and Communications at Lancaster University. Her research interests include designing tools and interactive systems to support highlevel skill acquisition and training such as creative and reflective thinking in design, autobiographical reasoning, emotional processing and spatial cognition. She has conducted design research on technologies for remembering, reflecting, sense-making and creative design thinking; and recently co-edited a Special Issue of Death Studies on Futures of Digital Death.

Will Odom (Simon Fraser University, Canada) is Assistant Professor in the School of Interactive Arts and Technology at Simon Fraser University, where he is CoDirector of the Everyday Design Studio. His research group takes an interdisciplinary, collaborative, creative, and design-oriented approach to Human-Computer Interaction research. He has a keen interest in exploring what it might mean to design and live with more enduring technologies in the context of everyday life. His work has explored how objects and

technologies support, and in some cases complicate, the peculiar ways in which we maintain relationships with the dead.

Kellie Morrissey (University of Limerick, Ireland) is a Lecturer in the School of Design at University of Limerick. A psychologist by training, her research interests lie at the intersection of health and politics in the design of digital objects in sensitive settings, including the use of virtual reality experiences in dementia, experience-centred approaches to designing for end of life, relational approaches to understanding the experiences of women undergoing menopause and online design processes for communities with chronic health issues.

Kyle Montague (Newcastle University, UK) is a Lecturer in Human-Computer Interaction based in Open Lab. His expertise and research interests include accessibility, wearable \& mobile interaction, and healthcare technologies. His work explores the design and configuration of digital technologies to support participation and inclusion of marginalised communities. 


\section{Pre-Workshop Plans}

A series of resources will be shared with accepted workshop participants well in advance of the conference.

These will comprise:

1. A set of conceptual descriptions of different theoretical approaches to bereavement and examples of design work created in response to end of life and bereavement (in order to help orientate participants to the topics and activities in the workshop);

\section{A design kit containing} short scenarios and provocation cards describing different end of life and bereavement narratives (and a series of short tasks to make notes in response and create a Pinterest board of things that relate to the narratives). All resources and Pinterest boards will be printed out in advance of the workshop for use in the design activities.
Nantia Koulidou (Northumbria University, UK) is a design researcher whose doctorate focused on digital jewellery in micro-transitions. Her methods are rooted in craft practices and participatory design. She is a Senior Research Associate on the RCUK Enabling Ongoingness project that is exploring how to enable people in early stages of dementia, in bereavement and at end-of-life to create content that will intersect dynamically with that created by their loved ones in future years.

Patrick Olivier (Monash University, Australia) is Professor of Human-Computer Interaction at Monash University. He coined the term Digital Civics, a crossdisciplinary endeavour that explores ways digital technologies can support new forms of civic participation. He has particular research interests in human-centred design methods and the design and social computing frameworks for coordinated action. He has a long track record in the participatory design of digital technologies and services for the health and wellbeing of older adults.

Wider Committee We will be supported by a larger committee of researchers who have agreed to provide guidance on the workshop's activities and outcomes (including reviewing submissions and promoting the workshop within their networks): Claire Craig (Sheffield Hallam University), Stephen Lindsay (Swansea University), Anne-Marie Piper (Northwestern University), Shaun Lawson (Northumbria University), Jon Rogers (University of Dundee), Niels Hendriks (LUCA School of Arts), Linnea Groot (Newcastle University), Luis Carvalho (Newcastle University), Wendy Moncur (University of Dundee), Francisco Nunes (Fraunhofer Portugal), John McCarthy (University
College Cork) and Frank Vetere (University of Melbourne).

\section{Community Partners}

Given the significant costs (environmental and otherwise) of a conference the scale of $\mathrm{CHI}$ we are seeking to find ways that conducting the workshop, can leverage local knowledge and expertise, of Hawaii in particular including the island's unique cultural configuration, and also make a small but positive contribution to organizations in the local community that are actively engaged in matters of end of life and beyond. We are currently in conversation with Hospice Hawaii - a non-profit, community-based organization that has provided care to tens of thousands of patients and their families - and with the Hawaii Psychological Association the leading resource for psychological health, research, and policy in Hawaii and touchpoint for advice concerning grieving.

\section{Workshop Structure}

We are open to a range of academic expertise and lived experience in relation to the workshop topic and aim for a supportive, creative and balanced set of activities. - During introductions, attendees will be invited to briefly share their backgrounds, research interests and (if comfortable) personal experiences of the topic. Participants will have seen each other's submissions in advance of the workshop and also participant responses to the pre-workshop tasks.

- Participants will break into three groups to discuss pre-workshop responses (both their own and other people's). Each group will present back the group's views and comments on things that have resonated with them.

- Following a coffee break community partners will 


Workshop Schedule
09:00 - 09:30 Welcome and
Icebreaker
09:30 - 10:30 Discussion
around pre-workshop
responses
10:30 - 11:00 Coffee break
11:00 - 12:00 Community
partners share experiences
from their organizations and
set challenges for participants
to consider
12:00 - 13:00 Design activity
part 1
13:00 - 14:00 Lunch
14:00 - 15:30 Design activity
part 2
15:30 - 16:00 Coffee break
16:00 - 17:00 Feedback to
larger group, bringing
together of important points
and future plans

present details about how their organization approaches end of life and bereavement and each set a challenge for participants to consider.

- In the first design activity each of the three groups will work with the set of provocation and scenario cards (that they have already seen pre workshop) in relation to the challenges set by community partners. Groups will develop a range of design ideas in response (design thinking ideation using sketching and discussion) and gain support and feedback from organizers and community partners.

- During a 'Working lunch' groups will go for food together to continue discussions around design ideas. - In the second design activity groups will focus in on one of their earlier design ideas and refine it considering interaction design, form, technology, methods of participation and ethical implications.

- Following a final coffee break groups will feedback to the larger group and organizers will bring together important points that have emerged and lead discussion on future plans. Throughout the day there will be an element of self-documentation by participants of the activities.

Please see sidebar for specific timings.

\section{Post Workshop Plans}

Workshop self-documentation, discussions and design outcomes will be collated into a document (with permissions) and shared with participants and the wider $\mathrm{CHI}$ community via the workshop url. We are currently in discussion with the Design for Health journal regarding a special issue focused on the topic of the workshop.

\section{Call for Participation: CHI 2019 Workshop on HCI at End of Life and Beyond.}

This one-day workshop will be held as part of the 2020 ACM SIGCHI Conference on Human Factors in Computing Systems, held in Honululu, HI, USA.

- Submission Deadline: 11 th February 2020

- Acceptance Notification: 28th February 2020

- Workshop Day: 25th or 26th April 2020

Death is an inevitable part of being alive. Approaching end of life, death and bereavement have implications for almost all users of digital technologies. We are living in an age with unforeseen capabilities to make both physical and digital "things" and where each person's life has an unavoidable associated trail of media and personal data. New opportunities to curate data and media to support others after our future death or to support ourselves in bereavement are substantial. The contexts of anticipating end of life and living with bereavement are changing as digital technologies become more embedded in our cultures. While it is easy to recognize that these opportunities for design are significant, and that the need is substantial and largely unaddressed, the barriers to working in a such sensitive context can be perceived by many $\mathrm{HCI}$ researchers as daunting.

In this workshop we aim to: develop discussion and design thinking around the opportunities for digital technologies; explore ethical concerns; and share design methodologies and methods to support the level of sensitivity and self-reflection required in this space. We invite attendees to submit 2-4 page statements of interest (in ACM Extended Abstract format) in .pdf format to jayne.wallace@northumbria.ac.uk 
Submissions can take one of several forms in relation to the workshop topic: standard academic positions; personal reflections from lived experience that have impacted the way that you research; or a design response/concept (a combination of sketches and written reflection/description akin to a DIS Pictorial). Please note, the workshop activities will involve design ideation activities and necessarily assume pre-event engagement with resources in order to meaningfully support the activities on the day.

Submissions will be reviewed by a committee of experts and selected on the basis of relevance to the workshop themes, quality of presentation, and potential to stimulate discussion. At least one author of each accepted submission must register for the workshop and at least one day of the main conference. For more information, please visit:

https://enablingongoingness.com/chi2020

\section{References}

[1] Madeline Balaam, R. Comber, R. Clarke, C. Windlin, A. Ståhl, K. Höök, and G. Fitzpatrick Emotion Work in Experience-Centered Design. CHI'19 ACM, 602.

[2] John Bowlby, and C. M. Parkes. "Separation and loss within the family." The child in his family 1. 1970.

[3] Sigmund Freud. "Mourning and melancholia." 1917.

[4] Glennys Howarth, 2011. The rebirth of death: continuing relationships with the dead. In Remember me Routledge, 29-44.

[5] Dennis Klass, P.R., Silverman and S. L. Nickman, eds. Continuing bonds: New understandings of grief. Taylor \& Francis, 1996.

[6] Dennis Klass and T. Walter, Processes of grieving: How bonds are continued. 2001.

[7] Elizabeth Kubler-Ross. "On Death and Dying' Macmillan." New York (1969).
[8] Michael Massimi, W. Odom, D. Kirk, and R. Banks. "HCI at the end of life: understanding death, dying, and the digital." CHI'10 pp. 4477-4480. ACM, 2010.

[9] Michael Massimi, W. Odom, R. Banks, and D. Kirk. "Matters of life and death: locating the end of life in lifespan-oriented hci research." CHI'11 pp. 987-996. ACM, 2011.

[10] Cosmin Munteanu, H. Molyneaux, W. Moncur, M. Romero, S. O'Donnell, and J. Vines. "Situational ethics" CHI'15 pp. 105-114. ACM, 2015.

[11] Paratene Ngata, 2005. Death, dying, and grief: A Maori perspective. Death and bereavement around the world 4, 29-38.

[12] Lisa P Nathan, A. Thieme, D. Tatar, and S. Branham, 2016. Disruptions, dilemmas and paradoxes: Ethical matter(s) in design research. Interacting with Computers, 1-9.

[13] Corina Sas, S. Whittaker, and J. Zimmerman. Design for Rituals of Letting Go: An Embodiment Perspective on Disposal Practices Informed by Grief Therapy. ACM Trans. Comput.-Hum. Interact. 23, no 4, Article 21 (August 2016), 37 pages.

[14] Corina Sas and A. Coman. Designing personal grief rituals: An analysis of symbolic objects and actions. Death studies 40, no. 9 (2016), 558-569.

[15] Corina Sas, M. Schreiter, M. Büscher, F. Gamba, and A. Coman. Futures of digital death: Past, present and charting emerging research agenda. Death Studies 43, no 7 (2019): 407-413.

[16] Margaret Stroebe, H. Schut. The dual process model coping with bereavement: Rationale and description. Death studies 23, (1999): 197-224.

[17] Piers Vitebsky, 1993. Dialogues with the dead: the discussion of mortality among Sora of eastern India.

[18] Jayne Wallace, J. Thomas, D. Anderson, P. Olivier. Mortality as framed by ongoingness in digital design. Des. Issues 34(1), 95-107 (2018).

[19] J. William Worden, Grief Counselling and Grief Therapy, Springer Publishing 1982. 